\title{
EVALUATION OF THE E-FUNCTION WHEN TWO OF THE UPPER PARAMETERS ARE EQUAL OR DIFFER BY AN INTEGER
}

\author{
SHAHWAR F. RAGAB
}

\section{Introduction.}

In a former paper [1] in this journal. I evaluated infinite series involving MacRoberts' $E$-functions, the definitions and properties of which are to be found in ([2]; pp; 348-352) and in ([3], pp; 203-206). The E-function is defined ([2], p. 409) thus: If $p \geq q+1$, then

$$
\begin{aligned}
E\left(p, \alpha_{r} ;, q ; \rho_{s}: z\right) & =\sum_{r=1}^{p}\left[\Pi_{s=1}^{p} \Gamma\left(\alpha_{s}-\alpha_{r}\right)\right]\left[\Pi_{t=1}^{q} \Gamma\left(\rho_{t}-\alpha_{r}\right)\right]^{-1} \\
& \Gamma\left(\alpha_{r}\right) Z^{\alpha_{r}} F\left\{\begin{array}{l}
\alpha_{r}, \alpha_{r}-\rho_{1}+1, \ldots, \alpha_{r}-\rho_{q}+1 ;(-1) p-q_{z} \\
\alpha_{r}-\alpha_{1}+1, \ldots, * \ldots, \alpha_{r}-\alpha_{\rho+1}
\end{array}\right\} \\
& =\sum_{r=1}^{p}\left[Z^{\alpha r} \sum_{n=0}^{\infty} \Gamma\left(\alpha_{r}+n\right) \frac{\Pi_{t=1}^{p} \Gamma\left(\alpha_{t}-\alpha_{r}-n\right)}{n ! \Pi_{s=1}^{q} \Gamma\left(\rho_{s}-\alpha_{r}-n\right)}(-z)^{n}\right],
\end{aligned}
$$

where $|\arg z|<\frac{1}{2}(p-q-1) \pi$, the prime in the product sign signifies that the factor for which $s=r$ is left and the asterisk in the $E$-function means that the parameter $\alpha_{r}-\alpha_{r}+1$ is omitted

If $p \leq q$, then ([2]; p. 352).

$$
E\left(p, \alpha_{r} ; q ; \rho_{s}: z\right)=\frac{\Gamma\left(\alpha_{1}\right) \ldots \Gamma\left(\alpha_{p}\right)}{\Gamma\left(\rho_{1}\right) \ldots \Gamma\left(\rho_{q}\right)} F\left\{\begin{array}{l}
\alpha_{1}, \ldots, \alpha_{p}, \frac{-1}{z} \\
\rho_{1}, \ldots, \rho_{q}
\end{array}\right\},
$$

Now, if two of the $\alpha^{\prime} s$ are equal or differ by integral values, some of the series on the right of (1) cease to exist. For instance, if $\alpha_{1}=\alpha+\ell, \alpha_{2}=\alpha$, where $\ell$ is a positive integer, the first two series are nom existent. Here it will be shown that they can be replaced by the expression

$$
\begin{aligned}
& (-1)^{\ell} z^{\alpha+\ell} \sum_{n=0}^{\infty} \frac{\Gamma(\alpha+\ell+n) \Pi_{t=1}^{p} \Gamma\left(\alpha_{t}-\alpha-\ell-n\right)}{n !(\ell+n) ! \Pi_{s=1}^{q} \Gamma\left(\rho_{s}-\alpha-\ell-n\right)} \Delta_{n} Z^{n} \\
& +z^{\alpha} \sum_{n=0}^{\ell-1} \frac{[\Gamma(\alpha+n)](\ell-n-1) ! \Pi_{t=3}^{p} \Gamma\left(\alpha_{t}-\alpha-n\right)}{n ! \Pi_{s=1}^{q}\left(\rho_{s}-\alpha-n\right)}(-z)^{n},
\end{aligned}
$$

Received. July 26, 1989.

Department of mathematics faculty of engineering Cairo university, Cairo (E.A.R.) Egypt. 
where

$$
\begin{aligned}
\Delta_{n}= & \psi(\ell+n)+\psi(n)-\psi(\alpha+\ell+n-1)-\log z \\
& \quad+\sum_{t=3}^{p} \psi\left(\alpha_{t}-\alpha-\ell-n-1\right)+\sum_{s=1}^{q} \psi\left(\rho_{s}-\alpha-\ell-n-1\right)
\end{aligned}
$$

and

$$
\psi(z)=\frac{d}{d z}\{\log \Gamma(z+1)\}=\frac{\Gamma^{\prime}(z+1)}{\Gamma(z+1)} .
$$

The proof of (3) will be given in $\S 2$, whils two subsidiary theorems will be stated and proved in $\S 3$. Also the value of $K_{0}(z)$, where $K_{0}(z)$ is the modified Bessel function of the second kind, will be derived in $\S 3$; while new infinite integrals will be deduced as particular cases in $§ 4$.

The following formulae will be required in the proofs: ([2]; p.141)

$$
\begin{aligned}
\psi(z+n) & =\psi(z)+\sum_{r=1}^{n} \frac{1}{z+r} \\
\psi(-z-1) & =\psi(z)+\pi \cot (\pi z) \\
& \psi(0)=-\gamma \\
& \psi(n)=\phi(n)-\gamma ;
\end{aligned}
$$

where

$$
\begin{aligned}
\gamma & =\lim _{m \rightarrow \infty}\left[\left(\frac{1}{1}+\frac{1}{2}+\ldots+\frac{1}{m}\right)-\log m\right], \\
\phi(n) & =\frac{1}{1}+\frac{1}{2}+\ldots+\frac{1}{n}, \quad \phi(0)=0
\end{aligned}
$$

If $m$ is a positive integer, then ([2], p.154, ex.5).

$$
\mathbb{\Pi}_{t=0}^{m-1}\left\{\Gamma\left(Z+\frac{t}{m}\right)\right\}=(2 \pi)^{\frac{1}{2} m-\frac{1}{2}} m^{\frac{1}{2}-m z} \Gamma(m z)
$$

Also ([2], p.207).

$$
K_{\mu}(z)=\frac{\pi}{2 \sin \mu \pi}\left\{I_{\mu}(z)-I_{\mu}(z)\right\}
$$

where

$$
I_{\mu}(z)=\sum_{n=0}^{\infty} \frac{1}{n ! \Gamma(n+\mu+1)}\left(\frac{z}{2}\right)^{\mu+2 n}
$$

If $m$ is a positive integer and if $R(k)>0$, then ([2], p.466 ex. 30).

$$
\int_{0}^{\infty} e^{-t} t^{k-1} E\left(p ; \alpha_{r} ; q, \rho_{s}: \frac{z}{t^{m}}\right) d t=m^{k-\frac{1}{2}}(2 \pi)^{\frac{1}{2}-\frac{1}{2} m} E\left(p+m, \alpha_{r}: q: \rho_{s}: \frac{z}{m^{m}}\right),
$$


where $\alpha_{p+\nu}=\frac{k+\nu-1}{m} ; \nu=1,2, \cdots, m-1$. If $x$ is real and positive and $R(m \pm \mu)>0$, then ([2]; p 395, ex. 109).

$$
4 \int_{0}^{\infty} t^{m-1} K_{\mu}(2 t) E\left(p, \alpha_{r} ; p ; \rho_{s}: \frac{x}{t^{2}}\right) d t=E\left(p+2 ; \alpha_{r} ; q ; \rho_{s}: x\right)
$$

where

$$
\alpha_{p+1}=\frac{1}{2}(m+\mu), \quad \alpha_{p+2}=\frac{1}{2}(m-\mu) .
$$

§2. Proof of the main theorem (3).

If $\alpha_{1}=\alpha+\ell, \alpha_{2}=\alpha+\epsilon$, where $\ell$ is zero or a positive integer and $\epsilon$ is small, the sum of the first two series on the right of (1), can be written

$$
\begin{gathered}
Z^{\alpha+\ell} \sum_{n=0}^{\infty} \frac{\Gamma(\alpha+\ell+n) \Gamma(-\ell-n+\epsilon) \Pi_{t=3}^{p} \Gamma\left(\alpha_{t}-\alpha-n-\ell\right)}{n ! \Pi_{s=1}^{q} \Gamma\left(p_{s}-\alpha-\ell-n\right)}(-z)^{n} \\
+Z^{\alpha+\epsilon} \sum_{n=0}^{\infty} \frac{\Gamma(\alpha+n+\epsilon) \Gamma(\ell-n-\epsilon) \Pi_{t=3}^{p} \Gamma\left(\alpha_{t}-\alpha-n-\epsilon\right)}{n ! \Pi_{s=1}^{q} \Gamma\left(\rho_{s}-\alpha-n-\epsilon\right)}(-z)^{n} . \\
=(-1)^{\ell} Z^{\alpha+\ell} \sum_{n=0}^{\infty} \frac{\Gamma(\alpha+\ell+n) \Pi_{t=3}^{p} \Gamma\left(\alpha_{t}-\alpha-\ell-n\right)}{n ! \Gamma(1+\ell+n-\epsilon) \Pi_{s=1}^{q} \Gamma\left(\rho_{s}-\alpha-\ell-n\right)}\left(\frac{\pi}{\sin \epsilon \pi}\right) Z^{n} \\
-(-1)^{\ell} Z^{\alpha+\epsilon} \sum_{n=\ell}^{\infty} \frac{\Gamma(\alpha+n+\epsilon) \Pi_{t=3}^{p} \Gamma\left(\alpha_{t}-\alpha-n-\epsilon\right)}{n ! \Gamma(1-\ell+n+\epsilon) \Pi_{s=1}^{q} \Gamma\left(\rho_{s}-\alpha-n-\epsilon\right)}\left(\frac{\pi}{\sin \epsilon \pi}\right) Z^{n} \\
+Z^{\alpha+t} \sum_{n=0}^{\ell-1} \frac{\Gamma(\alpha+n+\epsilon) \Gamma(\ell-n-t) \Pi_{t=3}^{p} \Gamma\left(\alpha_{t}-\alpha-n-\epsilon\right)}{n ! \Pi_{s=1}^{q} \Gamma\left(\rho_{s}-\alpha-n-\epsilon\right)}(-Z)^{n} .
\end{gathered}
$$

The limit when $\epsilon \longrightarrow 0$ of the first two terms in the last expression is obtained by removing the factor $\frac{\pi}{\sin \epsilon \pi}$, then differentiating with respect to $\epsilon$, and finally making $\epsilon \longrightarrow 0$. On replacing $n$ by $\ell+n$ in the second series of the last expression, formula (3) is obtained. Thus (3) is proved.

\section{§3. Derivation of $K_{0}(z)$.}

The first subsidiary theorem to be proved is

$$
K_{\mu}(z)=\frac{1}{4 \pi} \sum_{i,-i} \frac{1}{L} E\left(1, \frac{1}{2} \mu, \frac{-1}{2} \mu ; e^{i \pi} \frac{z^{2}}{4}\right)
$$

where $K_{\mu}(z)$ is the modified Bessel function of the second kind defined by (11) and (12) and the symbol $\Sigma_{i,-i}$ means that in the expression following it $i$ is to be replaced by $-i$ and the two expressions are to be added. 
To prove (15), expand the $E$-function on the right of (15) by means of (1), substract the corresponding terms and so obtain (15) by a second application of (12). To find the value of $K_{0}(z)$, put $\mu=0$ in (15) so getting

$$
K_{0}(z)=\frac{1}{4 \pi} \sum_{i,-i} \frac{1}{i} E\left(0,0,1:: \frac{z^{2}}{4}\right) .
$$

From (3) with $\alpha=\ell=0, p=3, q=0$, this becomes,

$$
\begin{aligned}
(4 i \pi) \cdot K_{0}(z) & =\sum_{\nu=0}^{\infty} \frac{\pi}{(\nu !)^{2} \sin \nu \pi}\left(\frac{z^{2}}{4}\right)^{\nu} \cdot(2 i \sin \pi \nu) \\
& {[2 \psi(\nu)-\psi(\nu-1)+\psi(-\nu)]+\sum_{\nu=0}^{\infty} \frac{\pi}{(\nu !)^{2} \sin \nu \pi}\left(\frac{z^{2}}{4}\right)^{\nu} } \\
& {\left[e^{-i \pi^{\nu}}\left(-i \pi+2 \log \left(\frac{z}{2}\right)-e^{i \pi \nu} \cdot\left(i \pi+2 \log \frac{z}{2}\right)\right]\right.} \\
& =\sum_{\nu=0}^{\infty} \frac{2 i \pi}{(\nu !)^{2}}[2 \psi(\nu)-\psi(\nu-1)+\psi(-\nu)]\left(\frac{z^{2}}{4}\right)^{\nu} \\
& +\sum_{\nu=0}^{\infty} \frac{\pi}{(\nu !)^{2} \sin \pi \nu}(2 i \sin \nu \pi) \\
& {\left[-i \pi(2 \cos \nu \pi)-2 \log \frac{z}{2}\right] \cdot\left(\frac{z^{2}}{4}\right)^{\nu} . }
\end{aligned}
$$

Now apply (5) and (7) and get.

$$
\begin{gathered}
4 i \pi K_{0}(z)=\sum_{\nu=0}^{\infty} \frac{2 i \pi}{(\nu !)^{2}}\left(\frac{z^{2}}{4}\right)^{\nu}[2 \phi(\nu)-2 \gamma] \\
-2\left(\log \frac{z}{2}\right) \cdot \sum_{\nu=0}^{\infty} \frac{2 i \pi}{(\nu !)^{2}}\left(\frac{z^{2}}{4}\right)^{\nu} . \\
K_{0}(z)=\sum_{\nu=0}^{\infty} \frac{1}{(\nu !)^{2}}\left(\frac{z^{2}}{4}\right)^{\nu} \phi(\nu)-\left[\gamma+\log \frac{z}{2}\right] I_{0}(Z)
\end{gathered}
$$

which is a known result ([2], p.268).

The second theorem to be proved in this section is

$$
\sum_{t=1}^{m-1} \psi\left(\frac{t}{m}-n-1\right)=m \phi(m n)-\phi(n)-(m-1) \gamma-m \log m ;
$$

where $m$ and $n$ are positive integres. 
To prove (17) take logarithms of both sides of (10), differentiate with respect to $Z$, so getting

$$
\sum_{t=1}^{m-1} \psi\left(z+\frac{t}{m}-1\right)=m \psi(m z-1)-m \log m-\psi(z) .
$$

Now apply (5), and get

$$
\sum_{i=1}^{m-1} \psi\left(z+\frac{t}{m}-1\right)=m \psi(-m z)-\psi(-z)-m \log m+\pi \cot \pi z-m \pi \cot (\pi m z) .
$$

But, when $z \longrightarrow-n, \pi \cot \pi z-m \pi \cot \pi(m z) \longrightarrow 0$.

From this (17) follows. Thus (17) is proved.

\section{§4. Evaluation of certain infinite integrals.}

We are now in a position to evaluate a large number of infinite new integrals by applying formula (3).

For example, if $m$ is a positive integer, $\mu=0$; then (14) gives

$$
\int_{0}^{\infty} \lambda^{m-1} K_{0}(2 \lambda) e^{-\frac{\lambda^{2}}{z}} d \lambda=\frac{1}{4} E\left(\frac{m}{2}, \frac{m}{2}:: z\right) .
$$

When $m=1$, from (3) with $\ell=0, \alpha=\frac{1}{2}, p=2, q=0$ this becomes

$$
\frac{1}{4} \sqrt{z} \sum_{0}^{\infty} \frac{\Gamma\left(\frac{1}{2}+n\right)}{(n !)^{2}} Z^{n}\left[2 \psi(n)-\psi\left(n-\frac{1}{2}\right)-\log z\right] .
$$

Now apply (7) and get

$$
\begin{aligned}
\int_{0}^{\infty} e^{-\frac{\lambda^{2}}{z}} K_{0}(2 \lambda) d \lambda & =\frac{1}{4} \sqrt{\pi z} \sum_{n=0}^{\infty} \frac{\left(\frac{1}{2} ; n\right)}{(n !)^{2}}\left[2 \phi(n)-\psi\left(n-\frac{1}{2}\right)\right] Z^{n} \\
& -\frac{1}{4} \sqrt{z \pi}(2 \gamma+\log z){ }_{1} F_{1}\left(\frac{1}{2} ; 1 ; z\right)
\end{aligned}
$$

When $m=2,(18),(3),(7)$ and (8) give

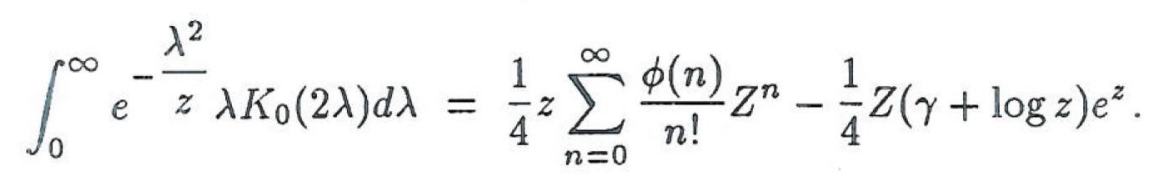

Again, if $m=1, \mu=0, \alpha_{1}=1$, then (14) gives

$$
\int_{0}^{\infty} \frac{K_{0}(2 \lambda)}{\left(\lambda^{2}+z\right)} d \lambda=\frac{1}{4 z} E\left(\frac{1}{2}, \frac{1}{2}, 1:: z\right) .
$$


From (3) with $\ell=0, \alpha_{1}=\alpha_{2}=\frac{1}{2}, \alpha_{3}=1, p=3, q=0$, this becomes

$$
\begin{aligned}
& \frac{1}{4 \sqrt{z}} \sum_{n=0}^{\infty} \frac{\Gamma\left(\frac{1}{2}+n\right) \Gamma\left(\frac{1}{2}-n\right)}{(n !)^{2}} z^{n}\left[2 \psi(n)-\log z+\psi\left(-n-\frac{1}{2}\right)-\psi\left(n-\frac{1}{2}\right)\right] \\
& -\frac{1}{4} \sum_{n=0}^{\infty}\left[\Gamma\left(-\frac{1}{2}-n\right)\right]^{2}(-z)^{n} .
\end{aligned}
$$

Now from (5), (6), (7), (8) and (9), this becomes.

$$
\begin{aligned}
& \int_{0}^{\infty} \frac{K_{0}(2 \lambda)}{\left(z+\lambda^{2}\right)} d \lambda=\frac{\pi}{2 \sqrt{z}} \sum_{n=0}^{\infty} \frac{(-z)^{n}}{(n !)^{2}} \phi(n)-\frac{\pi}{4 \sqrt{z}}(2 \gamma+\log z) \\
& \stackrel{J}{0}(2 \sqrt{z})+\pi_{1} F_{2}\left(1 ; \frac{3}{2}, \frac{3}{2} ;-z\right)
\end{aligned}
$$

where $\tilde{n}(z)$ is the Bessel function of the first kind. Also, if $m=2, \mu=0, p=1$, with $\alpha_{1}=\frac{1}{2}, q=0$; then (14) gives

$$
\int_{0}^{\infty} \frac{\lambda K_{0}(2 \lambda)}{\sqrt{z+\lambda^{2}}} d \lambda=\frac{1}{4 \sqrt{\pi z}} E\left(1,1, \frac{1}{2}:: z\right)
$$

From (3) with $\ell=0, \alpha=1, p=3, q=0, \alpha_{3}=\frac{1}{2}$; this becomes

$$
\begin{aligned}
\int_{0}^{\infty} \frac{\lambda K_{0}(2 \lambda)}{\sqrt{z+\lambda^{2}}} d \lambda= & \frac{\sqrt{z}}{2}(\gamma+\log z) \frac{\sin (2 \sqrt{z})}{2 \sqrt{z}}-\frac{\sqrt{z}}{2} \\
& \cdot \sum_{n=0}^{\infty} \frac{\phi(n)-\psi\left(n+\frac{1}{2}\right)}{n !\left(\frac{3}{2} ; n\right)}(-z)^{n}+\frac{\pi}{4} \cos (2 \sqrt{z})
\end{aligned}
$$

Again, if $m$ is any positive integer, then (13) with $k=1$ becomes

$$
\int_{0}^{\infty} \frac{e^{-t}}{z+t^{m}} d t=(2 \pi)^{\frac{1}{2}}-\frac{m}{2} m-\frac{1}{2} z^{-1} E\left(1,1, \frac{1}{m}, \frac{1}{m}, \frac{2}{m}, \ldots, \frac{m-1}{m}:: \frac{z}{m^{m}}\right)
$$

From (3) with $\ell=0, \alpha=1, p=1+m, q=0$, this becomes. 


$$
\begin{aligned}
\int_{0}^{\infty} \frac{e^{-t}}{z+t^{m}} d t & =(2 \pi)^{\frac{1}{2}}-\frac{1}{2} m \frac{1}{2}-m \sum_{n=0}^{\infty} \frac{1}{(n !)}\left[\Gamma\left(\frac{t}{m}-1-n\right)\right] \\
& \cdot\left(\frac{z}{m^{m}}\right)^{n}\left[\psi(n)-\log \left(\frac{z}{m^{m}}\right)+\sum_{t=1}^{m-1} \psi\left(\frac{t}{m}-2-n\right)\right] \\
& +(2 \pi)^{\frac{1}{2}}-\frac{m}{2} m-\frac{1}{2} z^{-1} \sum_{t=1}^{m-1}\left(\frac{z}{m^{m}}\right)^{\frac{t}{m}} \sum_{n=0}^{\infty} \frac{1}{n !} \Gamma\left(\frac{t}{m}+n\right)\left[\Gamma\left(1-\frac{t}{m}-n\right)^{2}\right. \\
& {\left[\Pi_{s=1}^{m=1} \Gamma\left(\frac{s-t}{m}-n\right)\right]\left(\frac{-z}{m^{m}}\right)^{n} . }
\end{aligned}
$$

Here apply (17) with $(n+1)$ in place of $n$ noting that

$$
m \phi(m n+m)-\phi(n+1)=\phi(m n+m-1)-\phi(n)
$$

then the last expression becomes

$$
\begin{aligned}
\int_{0}^{\infty} \frac{e^{-t}}{z+t^{m}} d t & \left.=\frac{(-1)^{m+1}}{m} \sum_{n=0}^{\infty} \frac{\left\{(-1)^{m+1} z\right\}^{n}}{(m n+m-1) !}[m \phi(m n+m-1)-m \gamma-\log z)\right] \\
& -\frac{\pi}{m z} \sum_{t=1}^{m-1} \frac{\left(-z^{\frac{1}{m}}\right)^{t}}{\sin \left(\frac{\pi t}{m}\right)} \sum_{n=0}^{\infty} \frac{\left\{(-1)^{m+1} z\right\}^{n}}{(m n+t-1) !}
\end{aligned}
$$

Many particular cases can be derived from the main theorems (21), (22) and (23) by specializing the values of the parameters $p, q$ and $m$. For example, if $m=1$, (23) becomes.

$$
\int_{0}^{\infty} \frac{e^{-t}}{z+t} d t=-(\gamma+\log z) e^{z}+\sum_{n=0}^{\infty} \frac{\phi(n)}{n !} Z^{n}
$$

\section{References}

[1] Ragab, S.F. "Infinite series of E-functions" Tamkang J. of Math. V.20, ..., 1989.

[2] MacRobert., T.M., "Functions of a complex variables Macmillon and Comp. London 1962.

[3] Erdelyi A., Magnus W., oberhettinger F. and Tricomi F., "Higher "Transcendental functions Vol. I. " Mcgrew Hill 1953.

Faculty of Engineering, Cairo University, Cairo (E.A.R.) Egypt. 